\title{
Immune Checkpoint Inhibitors and Novel Agents in the Treatment of Metastatic Urothelial Cancer- Current Status and Future Perspectives
}

\author{
An Expert Interview with Petros Grivas
}

Genitourinary Cancers Program, University of Washington, Seattle, WA,USA

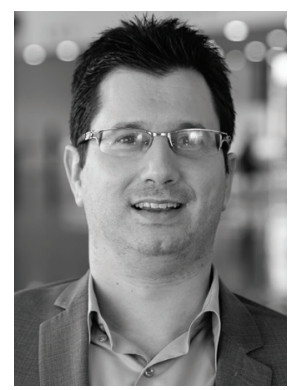

\section{Petros Grivas}

Petros Grivas, MD, PhD is a board-certified Medical Oncologist at Seattle Cancer Care Alliance, WA, USA. He also serves as Clinical Director of the University of Washington School of Medicine's Genitourinary Cancers Program and is a University of Washington Associate Professor. Dr Grivas is considered an international expert in genitourinary cancers. He earned his MD and PhD at the University of Patras in Greece and is board-certified in Medical Oncology and Internal Medicine. He lectures internationally, reviews for scientific journals, pursues community outreach, leads translational and clinical studies and publishes novel research. Dr Grivas has participated in clinical trials leading to United States Food and Drug Administration approval of new drugs for urothelial cancer.

\section{Keywords}

Immune checkpoint inhibitors, urothelial cancer, atezolizumab, pembrolizumab, combination therapy

Disclosure: Petros Grivas has acted as a consultant for AstraZeneca, Bayer, Biocept, Bristol-Myers Squibb, Clovis Oncology, Driver, EMD Serono, Exelixis, Foundation Medicine, Genentech, Genzyme, GlaxosmithKline, Heron Therapeutics, Janssen, Merck \& Co., Mirati Therapeutics, Pfizer, Roche, Seattle Genetics, QED Therapeutics. $\mathrm{He}$ has been involved in educational programs with Bristol-Myers Squibb, Genentech. Research funding to his institution has been provided by AstraZeneca, Bayer Genentech/Roche, Merck \& Co., Mirati Therapeutics, Oncogenex, Pfizer, Clovis Oncology, Bavarian Nordic, Immunomedics, Debiopharm and Bristol-Myers Squibb.

Acknowledgments: Medical writing assistance was provided by Katrina Mountfort of Touch Medical Media, and supported by Touch Medical Media.

Review Process: This is an expert interview and as such, has not undergone the journal's standard peer review process.

Compliance with Ethics: This article is an opinion piece and does not report on new clinical data, or any studies with human or animal subjects performed by any of the authors.

Authorship: The named author meets the Internationa Committee of Medical Journal Editors (ICMJE) criteria for authorship of this manuscript, takes responsibility for the integrity of the work as a whole, and has given final approval to the version to be published.

Received: October 23, 2019

Accepted: November 15, 2019

Citation: Oncology \& Hematology Review (US). 2019; 15(2):68-70

Corresponding Author: Petros Grivas, Associate Professor, Clinical Director of Genitourinary Cancers Program University of Washington, Seattle, WA, 98115 USA.

E: pgrivas@uw.edu

Support: No funding was received in

the publication of this article.
A dvanced (locally advanced/unresectable or metastatic) urothelial cancer (UC) is an incurable disease with a poor prognosis.' Platinum-based chemotherapy is the first-line treatment for most patients and, until recently, patients with advanced UC that has progressed after platinum-based chemotherapy had limited treatment options. ${ }^{2}$ In recent years, improved understanding of molecular mechanisms underlying the pathogenesis of UC has enabled considerable therapeutic advances, including the use of immune checkpoint inhibitors, which transformed the management of this disease. ${ }^{3}$

In an expert interview conducted at the European Society for Medical Oncology (ESMO) Congress, which was held on September 27-October 1, 2019 in Barcelona, Spain, Dr Grivas discussed the use of immune checkpoint inhibitors in UC, as well as examples of ongoing studies investigating novel agents and combined therapeutic approaches.

\section{Q. How have immune checkpoint inhibitors changed the treatment landscape for metastatic urothelial cancer?}

The evolving landscape for metastatic UC is very exciting, particularly with immune checkpoint inhibitors. We now have five immune checkpoint inhibitors that are approved by the US Food and Drug Administration (FDA) for platinum-refractory advanced UC: pembrolizumab, atelolizumab, nivolumab, durvalumab and avelumab. Pembrolizumab has level 1 evidence in this treatment setting, based on the KEYNOTE-045 trial. ${ }^{4}$ Two of these agents, atezolizumab and pembrolizumab, have also been approved in the front-line setting for patients who are not fit enough for cisplatin and have tumors with high expression of programmed death-ligand 1 (PD-L1), based on companion corresponding assay.

In the United States, atezolizumab and pembrolizumab can be used in any patient who is not suitable for any platinum (cisplatin and carboplatin) chemotherapy, regardless of tumor tissue PD-L1 expression, based on the FDA label. It is important to note that a plethora of combination clinical trials are trying to build on this success with immune checkpoint inhibitors plus chemotherapy, antibody drug conjugates, targeted therapies, anti-angiogenesis agents, vaccines, cytokines, etc. ${ }^{3}$ The successful implementation of immune checkpoint inhibitors represents a significant change in the treatment paradigm, with hopefully more to come in the future. 


\section{Q. What progress has been made in identifying biomarkers of response to immune checkpoint inhibitors in urothelial cancer?}

Biomarkers are considered the 'Holy Grail', not only in UC, but across cancer types in general. Some biomarkers, such as tumor tissue PD-L1, have clinical value in the frontline setting in cisplatin-unfit patients, as discussed above. There are a number of promising putative biomarkers, such as tumor mutational burden (TMB), immune cell infiltration, tumor microenvironment (e.g. transforming growth factor beta [TGF- $\beta$ ] and epithelial-mesenchymal transition [EMT] signatures), T cell density, clonality and diversity, mutations in DNA damage response genes, molecular subtypes based on gene expression profiling, circulating tumor DNA, Tregs, myeloid-derived suppressor cells, etc. ${ }^{5}$ These all need to be validated, ideally in prospective clinical trials, before they can be incorporated into clinical practice. In addition to other potential biomarkers, TMB appears very promising, but has no clinical utility at the moment in UC and warrants validation studies, as well as standardization of methods/assays, selection of optimal cut-off points, etc. ${ }^{5,6}$ Many studies, for example PREVAIL (NCT03788746), are prospectively evaluating biomarkers in patients with advanced UC.

\section{Q. What is the potential for combined treatment approaches using programmed cell death 1 (PD-1/ PD-L1) and cytotoxic T-lymphocyte-associated protein 4 (CTLA-4) inhibitors in urothelial cancer?}

The combination of CTLA-4 and PD-1 or PD-L1 inhibitors has been tested in different tumor types, for example kidney cancer and melanoma.? In platinum-refractory advanced UC, the combination of ipilimumab and nivolumab showed an overall response rate of $38 \%$, and was as high as $58 \%$ in patients with high PD-L1 status in the tumor tissue, based on CHECKMATE 032 trial. ${ }^{8}$ That data is not practice-changing yet but there are three relevant large randomized Phase III trials that are ongoing in the frontline setting. The DANUBE trial (NCT02516241) is comparing the combination of durvalumab and tremelimimab or durvalumab alone to platinum-based chemotherapy. ${ }^{9}$ The CHECKMATE 901 study (NCT03036098) is comparing the combination of ipilimumab and nivolumab to platinum-based chemotherapy. The NILE trial (NCT03682068) is comparing tremelimumab and durvalumab plus platinum-based chemotherapy to chemotherapy/durvalumab or chemotherapy alone. In my opinion, these trials are going to help define the utility of combination of CTLA-4 and PD-1/PD-L1 inhibition in advanced UC and also help inform the development of biomarkers intended to select patients for the right treatment. Other trials are looking at ipilimumab and nivolumab in the neoadjuvant setting, for example the NABUCCO trial. Results from NABUCCO were recently presented at the 2019 European Society for Medical Oncology (ESMO) Meeting. Data from this trial is very interesting and supports further studies, but is not practice-changing yet.

For context, it is also noteworthy that two large three-arm Phase III trials (IMvigor130 [NCT02807636] and Keynote-361 [NCT02853305]) are evaluating the combination of chemotherapy plus atezolizumab or pembrolizumab in the first line setting of advanced UC, respectively. Initial results for the IMvigor130 trial were reported at the 2019 ESMO meeting, with longer followup needed to further assess overall survival and potential impact on clinical practice. ${ }^{10}$ The two switch maintenance trials are also relevant. Interesting results from the Phase II HCRN trial were presented at the 2019 ASCO meeting, while the large Phase III Javelin Bladder 100 trial (NCT02603432) has not been reported on yet. ${ }^{11}$ Results from all the above trials will generate important data that will inform the treatment landscape in the near future.

\section{Q. How can we best manage toxicity from immune checkpoint inhibitors?}

We have to understand more and do a better job of educating patients, caregivers and healthcare providers on early recognition and prompt management of immune-related adverse events (AEs). At the University of Washington and Seattle Cancer Care Alliance where I practice, we have formed the Immunotherapy-Related Adverse Event Tumor Board where we can discuss such cases. We continue to learn how to promptly recognize patients who have early subtle symptoms suggesting significant toxicity from immune checkpoint inhibitors and how to treat them in a timely and proper manner. We can then utilize data to inform future clinical decisions by building databases, registries and tumor boards, and therefore collectively develop more experience. In theory, any part of the body can be a target of an overactive immune system, and while AEs usually happen early in treatment, they can arise at any time, and less commonly even after treatment completion. Immunotherapy-related AEs are more common with combination therapies. ${ }^{12}$ We also need to encourage patients to report them, as many AEs are under-reported. Through a collaborative effort involving tumor boards, registries and clinical studies, we can develop more data to add to current publications addressing the optimal management of $A E S,{ }^{13}$ and evolve an infrastructure to recognize AEs which can, in rare cases, be very severe..$^{14-16}$

\section{Q. Which novel agents are in clinical development for metastatic urothelial cancer?}

There are several promising agents in development for UC, and I will give you a few examples rather than an exhaustive list. These include targeted therapies, for example erdafitinib, which received accelerated approval by the FDA for patients with previously treated (with platinum chemotherapy) metastatic bladder cancer harboring activating mutations or fusions in fibroblast growth factor receptor (FGFR)3 or FGFR2. This is a very promising agent, and is being investigated in an ongoing Phase III study (NCT03390504) comparing erdafitinib to either chemotherapy or pembrolizumab. A previous Phase II trial showed a very promising overall response rate of $30-40 \% .{ }^{17} \mathrm{~A}$ number of other promising FGFR inhibitors are being tested in clinical trials (e.g. NCT03834220), mostly in advanced disease; however, infigratinib and INCB054828 are being assessed also in the adjuvant setting.

Antibody-drug conjugates (ADC) such as enfortumab-vedotin, which targets nectin-4, are also under investigation. In a recent clinical trial, enfortumab-vedotin showed an impressive overall response rate of about $40 \%$ in pretreated patients with advanced $U \mathrm{CC}^{18}$ and has received a breakthrough designation by the FDA, while a Phase III trial is ongoing (NCT03474107). Moreover, very impressive efficacy data was presented at the 2019 ESMO Meeting for the combination of pembrolizumab and enfortumab-vedotin in the single arm EV-103 Phase $\mathrm{lb}$ trial in the first line setting of 45 cisplatin-unfit patients. These results support further, larger trials assessing this combination. Preliminary data from the TROPHY-01 Phase II trial was also presented at the 2019 ESMO meeting for the ADC sacituzumab-govitecan, with an impressive overall response rate approaching $30 \%$ in heavily pretreated patients after many lines of therapy. ${ }^{19}$ Other promising agents include trastuzumab-deruxtecan (DS8201) and RC48-ADC, both targeting human epidermal growth factor receptor 2 (HER-2), among other compounds. ${ }^{20,21}$ 
Additional promising targeted therapies include anti-angiogenesis and PARP inhibitors (e.g. NCT03898180; NCT03397394), which are now being investigated also in combination regimens. ${ }^{22}$

Combination approaches involving immune checkpoint inhibitors and other immune modulators, cytokines (e.g. NKTR-214, interleukin-7), vaccines (e.g. NCT03628716), chemotherapy (e.g. NCT03924856), radiation (e.g. NCT03775265; NCT03486197), anti-angiogenetic and targeted therapies, epigenetic therapies, ADC (e.g. NCT03523572) among others, are also in progress. The BISCAY Phase Ib study of durvalumab in combination with several targeted agents was presented at the 2019 ESMO meeting and generated very interesting hypotheses despite not informing clinical practice. ${ }^{23}$ Together with biomarker development, the above and other clinical trials are likely to give interesting results and possibly impact the evolving treatment landscape of UC in the future.
1. National Cancer Institute. SEER cancer stat facts: Bladder cancer, 2019. Available at: https://seer.cancer.gov/statfacts/html/urinb. html (accessed November 21, 2019).

2. Mar N, Dayyani F. Management of urothelial bladder cancer in clinical practice: real-world answers to difficult questions. J Onco Pract 2019:15:421-8.

3. Alhalabi $\mathrm{O}$, Shah $\mathrm{AY}$, Lemke EA, et al. Current and future landscape of immune checkpoint inhibitors in urothelial cancer. Oncology (Williston Park). 2019;33:11-8.

4. Bellmunt J, de Wit R, Vaughn DJ, et al. Pembrolizumab as secondine therapy for advanced urothelial carcinoma. N Eng/ J Med. 2017;376:1015-26.

5. Lavoie JM, Black PC, Eigl BJ. Predictive biomarkers for checkpoint blockade in urothelial cancer: A systematic review. J Urol. 2019;202:49-56

6. Yarchoan M, Albacker LA, Hopkins AC, et al. PD-L1 expression and tumor mutational burden are independent biomarkers in most cancers. JCl Insight. 2019;4:pii:126908.

7. Rotte A. Combination of CTLA-4 and PD-1 blockers for treatment of cancer. J Exp Clin Cancer Res. 2019;38:255.

8. Sharma P Siefker-Radtke A de Braud F et al. Nivolumab alone and with ipilimumab in previously treated metastatic urothelial carcinoma: CheckMate 032 nivolumab $1 \mathrm{mg} / \mathrm{kg}$ plus ipilimumab $3 \mathrm{mg} / \mathrm{kg}$ expansion cohort results. J Clin Oncol. 2019;37:1608-16.

9. Baldini $C$, Champiat $S$, Vuagnat $P$, et al. Durvalumab for the management of urothelial carcinoma: A short review on the emerging data and therapeutic potential. Onco Targets Ther.
2019;12:2505-12.

10. Grande E, Galsky M, Arranz Arija JA, et al. IMvigor130: efficacy and safety from a Phase 3 study of atezolizumab (atezo) as monotherapy or combined with platinum-based chemotherapy (PBC) vs placebo + PBC in previously untreated locally advanced or metastatic urothelial carcinoma (mUC). LBA14_PR: Presented at: European Society for Medical Oncology (ESMO) Congress, Barcelona, Spain, September 27-October 1, 2019.

11. Galsky MD, Pal SK, Mortazavi A. Randomized double-blind phase II study of maintenance pembrolizumab versus placebo after first-line chemotherapy in patients (pts) with metastatic urothelial cancer (mUC): (Suppl 15):4504.

12. Winer $\mathrm{A}$, Bodor JN, Borghaei $\mathrm{H}$. Identifying and managing the adverse effects of immune checkpoint blockade. I Thorac Dis. 2018;10(Suppl 3):S480-9.

13. Nagai H, Muto M. Optimal management of immune-related adverse events resulting from treatment with immune checkpoin inhibitors: a review and update. Int I Clin Oncol. 2018:23:410-20.

14. Thompson JA, Schneider BJ, Brahmer J. Management of immunotherapy-related toxicities, version 1.2019. J Nat/ Compr Canc Netw. 2019:17:255-89.

15. Kennedy LC, Bhatia S, Thompson JA. Preexisting autoimmune disease: implications for immune checkpoint inhibitor therapy in solid tumors. J Nat/ Compr Canc Netw. 2019;17:750-7.

16. Naidoo J, Zhang J, Lipson EJ. A multidisciplinary toxicity team for cancer immunotherapy-related adverse events. J Nat compr
Canc Netw. 2019;17:712-20

17. Loriot $Y$, Necchi A, Park SH, et al. Erdafitinib in locally advanced or metastatic urothelial carcinoma. N Eng/ J Med. 2019;381:338-48.

18. Rosenberg JE, O'Donnell PH, Balar AV et al Pivotal trial of enfortumab vedotin in urothelial carcinoma after platinum and anti-programmed death 1/programmed death ligand 1 therapy. J Clin Oncol. 2019;37:2592-600

19. Tagawa ST, Faltas BM, Lam ET, et al. Sacituzumab govitecan (IMMU-132) in patients with previously treated metastatic urothelial cancer (mUC): results from a phase I/II study, Presented at: European Society for Medical Oncology (ESMO) Congress, Barcelona, Spain, September 27-October 1, 2019.

20. Iwata $H$, Tamura K, Doi T, et al. Trastuzumab deruxtecan (DS 8201a) in subjects with HER2-expressing solid tumors: Long-term results of a large phase 1 study with multiple expansion cohorts. J Clin Oncol. 2019;36(Suppl 15):2501.

21. Sheng X, Zhou A, Yao X. A phase II study of RC48-ADC in HER2 positive patients with locally advanced or metastatic urothelia carcinoma. J Clin Oncol. 2019;37(Suppl 15):4509.

22. Criscuolo D, Morra F, Giannella R, et al. New combinatorial strategies to improve the PARP inhibitors efficacy in the urothelial bladder cancer treatment. J Exp Clin Cancer Res. 2019:38:91.

23. Powles TB, Balar A, Bravis G, et al. An adaptive, biomarker directed platform study in metastatic urothelial cancer (BISCAY) with durvalumab in combination with targeted therapies. Ann Oncol. 2019:30(Supp 5):V356-V402. 